\title{
Bur open The coronary CT angiography vision protocol: a prospective observational imaging cohort study in patients undergoing non-cardiac surgery
}

To cite: Sheth T, Butler C, Chow B, et al. The coronary CT angiography vision protocol: a prospective observational imaging cohort study in patients undergoing non-cardiac surgery. BMJ Open 2012;0:e001474. doi:10.1136/bmjopen-2012001474

- Prepublication history and additional material for this paper are available online. To view these files please visit the journal online (http://dx.doi.org/10.1136/ bmjopen-2012-001474).

Received 18 November 2011 Accepted 18 June 2012

This final article is available for use under the terms of the Creative Commons Attribution Non-Commercial 2.0 Licence; see http://bmjopen.bmj.com

For numbered affiliations see end of article

Correspondence to Dr Tej Sheth; shetht@mcmaster.ca

\section{ABSTRACT}

Introduction: At present, physicians have a limited ability to predict major cardiovascular complications after non-cardiac surgery and little is known about the anatomy of coronary arteries associated with perioperative myocardial infarction. We have initiated the Coronary CT Angiography (CTA) VISION Study to (1) establish the predictive value of coronary CTA for perioperative myocardial infarction and death and (2) describe the coronary anatomy of patients that have a perioperative myocardial infarction.

Methods and analysis: The Coronary CTA VISION Study is prospective observational study. Preoperative coronary CTA will be performed in 1000-1500 patients with a history of vascular disease or at least three cardiovascular risk factors who are undergoing major elective non-cardiac surgery. Serial troponin will be measured 6-12 h after surgery and daily for the first 3 days after surgery. Major vascular outcomes at 30 days and 1 year after surgery will be independently adjudicated. Ethics and dissemination: Coronary CTA results in a measurable radiation exposure that is similar to a nuclear perfusion scan (10-12 mSV). Treating physicians will be blinded to the CTA results until 30 days after surgery in order to provide the most unbiased assessment of its prognostic capabilities. The only exception will be the presence of a left main stenosis $>50 \%$. This approach is supported by best available current evidence that, excluding left main disease, prophylatic revascularisation prior to non-cardiac surgery does not improve outcomes. An external safety and monitoring committee is overseeing the study and will review outcome data at regular intervals. Publications describing the results of the study will be submitted to major peer-reviewed journals and presented at international medical conferences.

\section{INTRODUCTION}

Globally over 200 million patients undergo major non-cardiac surgery annually. Despite

\section{ARTICLE SUMMARY}

Article focus

This study protocol has two primary objectives.

- To establish the predictive value of coronary CT angiography for perioperative myocardial infarction and death

- To describe the preoperative coronary anatomy of patients that have a perioperative myocardia infarction.

Key messages

- Coronary CT angiography may be a valuable tool for risk stratification prior to non-cardiac surgery but needs to be evaluated in a prospective study.

Strengths and limitations of this study

- Large multicentre blinded imaging study with independent adjudication of clinical outcomes.

Recruitment targeted to a population at intermediate to high risk of peri-operative ischaemia.

the benefits of surgery, annually over 5 million non-cardiac surgery patients will suffer a cardiovascular death or non-fatal myocardial infarction in the first 30 days after surgery. ${ }^{1}$

\section{Limited capacity to predict major perioperative ischemic events}

Accurate risk estimation is important to allow patients and physicians to make informed choices about the appropriateness of surgery and to inform perioperative management (eg, anaesthetic approach). Risk prediction based on clinical risk factors and functional capacity is suboptimal. ${ }^{2}$ This is probably because many patients are inactive for substantial periods of time prior to their non-cardiac surgery (eg, orthopaedic, vascular and oncology 
patients) due to their underlying surgical condition, and as such, many patients with substantial coronary artery disease (CAD) may not have experienced any suggestive symptoms.

In an attempt to improve preoperative risk prediction, some patients undergo non-invasive cardiac stress tests (eg, stress echocardiography and nuclear scintigraphy imaging) prior to non-cardiac surgery. ${ }^{3}$ A recent meta-analyses evaluating these two tests demonstrated, however, that they have only moderate negative likelihood ratios (stress echocardiography 0.23 and stress perfusion imaging 0.44), and that more than a third of the patients who suffered a major perioperative cardiovascular event had a negative preoperative test result. ${ }^{4}$ These data represent likely a best-case scenario because most of the studies have not assessed whether these non-invasive cardiac stress tests provide independent prognostic information beyond known clinical variables. The few studies that have undertaken multivariable regression analysis provide unreliable estimates because they did not include all the known independent clinical variables or the analysis had too few events for the number of variables assessed. ${ }^{5-9}$

\section{Mechanisms of perioperative ischemic events}

Although perioperative myocardial infarction is the most common major perioperative cardiac complication, little is known about its pathophysiology. ${ }^{3}$ Understanding the pathophysiology of perioperative myocardial infarction is important to help inform which potential prophylactic interventions and acute management interventions should be evaluated in randomised controlled trials to improve the outcome of patients undergoing noncardiac surgery.

A commonly proposed mechanism of perioperative myocardial infarction relates to myocardial oxygen supply demand mismatch. Fluid shifts, catecholamine surges, hypotension, anaemia and hypoxia can occur during and after major non-cardiac surgery and transiently increase myocardial oxygen demand. ${ }^{3}$ In coronary vessels with high grade stenoses or occlusions, the supply response may be limited, resulting in supplydemand mismatch myocardial ischaemia or infarction. An additional or alternative mechanism of perioperative myocardial infarction is that the acute stress of surgery and mechanical tissue injury induces a hypercoagulable state that increases the risk of coronary thrombus formation at the site of a fissured plaque or with low coronary flow.

\section{Rationale for use of coronary CTA prior to non-cardiac surgery}

Coronary CTA may have several advantages for risk stratification prior to non-cardiac surgery. First, coronary CTA does not require exercise or pharmacological stress to detect CAD and therefore is well suited to the vascular and orthopaedic surgical populations who often cannot exercise or take the necessary pharmacological agents (eg, patients with asthma). Second, coronary CTA can exclude obstructive CAD very reliably as it has a high sensitivity for the detection of coronary stenosis. ${ }^{10-13}$

Third, coronary CTA is a very sensitive modality for the detection of high-risk coronary anatomy (left main disease or three or two vessel disease including the proximal left anterior descending (LAD) artery) as coronary CTA can visualise these large proximal vessels very well. ${ }^{13}$ Fourth, coronary CTA is the only non-invasive modality that can detect non-obstructive atherosclerosis, ${ }^{14}$ and some of our research (eg, POISE) ${ }^{15}$ offers clues that non-obstructive CAD may be responsible for a substantial proportion of the perioperative myocardial infarctions that occur in the non-cardiac surgery setting through plaque rupture and thrombosis.

Finally, coronary CTA can provide a comprehensive anatomic characterisation of the coronary arteries prior to surgery, and this has substantial potential to shed important insight into the extent of preoperative coronary atherosclerosis in culprit vessels that are associated with perioperative myocardial infarction.

\section{OBJECTIVES OF THE CORONARY CTA VISION STUDY}

The Coronary CTA VISION Study is a prospective cohort study that will examine patients with, or at risk of, atherosclerotic disease who are undergoing non-cardiac surgery in order to determine: if preoperative coronary CTA has additional predictive value, beyond clinical variables, for the occurrence of major perioperative cardiac events (ie, cardiovascular death and non-fatal myocardial infarction) at 30 days after surgery; and the underlying coronary anatomy associated with perioperative myocardial infarction. The Coronary CTA VISION Study is a substudy of the VISION Study. The Vascular events In non-cardiac Surgery patients cOhort evaluatioN (VISION) Study is a 40000 patient international prospective cohort study that we are currently undertaking, and this study is evaluating perioperative vascular complications in patients undergoing non-cardiac surgery.

\section{METHODS}

\section{Study design}

The Coronary CTA VISION Study is a prospective observational study of coronary CTA performed in patients prior to non-cardiac surgery.

\section{Study population}

The investigators will consider all patients undergoing elective non-cardiac surgery for enrolment. Boxes 1 and 2 present the study's inclusion and exclusion criteria.

\section{Clinical data collection}

After obtaining written informed consent, research personnel will interview and examine patients and review 


\section{Box 1 Inclusion criteria}

- All patients undergoing elective non-cardiac surgery are eligible if

- They are $\geq 45$ years of age.

- They require at least an overnight hospital admission after surgery.

- They are undergoing one of the following surgeries

- Orthopaedic (major joint arthroplasty)

- Vascular

- Thoracic surgery (pneumonectomy, lobectomy, other thoracic-wedge lung resection, mediastinal tumor resection, major chest wall resection)

- Major abdominal surgery (partial or total colectomy, stomach surgery, visceral resection, cytoreductive surgery, radial hysterectomy and radical prostatectomy)

- Major organ transplantation (kidney, liver, lung).

- They have enough time prior to non-cardiac surgery to obtain a coronary CTA study.

- They fulfill one of the following additional criteria:

- History of coronary artery disease

- History of peripheral vascular disease

- History of stroke

- History of a physician diagnosis of congestive heart failure; OR

- Any three of the following six risk factors: (a) diabetes and currently on an oral diabetic drug or insulin therapy, (b) age $\geq 70$ years, (c) history of smoking within 2 years of surgery, (d) history of treatment for hypercholesterolemia, (e) history of a transient ischaemic attack or ( $f$ ) a history of hypertension.

\section{Box 2 Exclusion criteria of the CTA VISION Study}

All patients are excluded if

- They are referred to invasive coronary angiography prior to non-cardiac surgery (coronary CTA will not provide information incremental to an invasive angiogram).

- They have had a prior percutaneous coronary intervention with stent implantation (due to limited ability of coronary CTA to evaluate stents).

- They have a creatinine clearance $<35 \mathrm{ml} / \mathrm{min}$ (to avoid risk of contrast nephrotoxicity in patients potentially at risk).

- They have a known contrast reaction.

- They are pregnant.

- They have persistent atrial fibrillation or $>2$ atrial or ventricular premature beats on a preoperative 12 lead ECG (suboptimal image quality results from irregular heart rhythms at cardiac CT due to difficulties with retrospective gating).

- They weight $>300$ lbs (obese patients have suboptimal image quality due to a poor contrast to noise ratio).

- They have an inability to achieve the required heart rate prior to coronary CTA despite medication (ie, a $\beta$-blocker or calcium channel blocker) if the patient will be scanned on a single source scanner they require a heart rate $<70 \mathrm{bpm}$ or a heart rate $<90 \mathrm{bpm}$ if the patient will be scanned on a dual source scanner.

- Patients who do not undergo non-cardiac surgery within 6 months of their coronary CTA. their charts to obtain information on patient characteristics that we are evaluating in the VISION Study to determine if they have potential independent predictors of major perioperative vascular events. These variables include risk factors, co-morbidities, medications, anaesthetic and surgical variables.

\section{CTA imaging protocol}

Once a patient is consented for the study, research personnel coordinate an appointment for the patient's preoperative coronary CTA scan. Personnel in the radiology department see patients prior to the coronary CTA scan and, when necessary, pretreat the patients with $\beta$-blockers (to achieve heart rate of 60 or less) and nitroglycerin (at a dose of $0.6-0.8 \mathrm{mg}$ administered sublingually) to optimise image quality. At centres with single source scanners, patients who continue to have a heart rate $70 \mathrm{bpm}$ or greater despite $\beta$-blockade are excluded from the study $(90 \mathrm{bpm}$ or greater with dual source scanners). In patients with asthma, personnel use a calcium channel blocker (diltazem $30-120 \mathrm{mg}$ ) is used to achieve heart rate control. A non-contrast scan is performed first. This scan is prospectively triggered at $75 \%$ of the $\mathrm{R}$ to $\mathrm{R}$ (RR) interval, with $0.4-0.625$ slice thickness (depending on scanner type) and $3 \mathrm{~mm}$ increments. Technicians next perform a contrast scan and inject contrast agent at a rate of $5.5-7 \mathrm{ml} / \mathrm{s}$ depending on body habitus of the patient being scanned. For an average patient, the dose of contrast is expected to be approximately $80 \mathrm{ml}$. The contrast scan is retrospectively gated or prospectively triggered with $0.4-0.625$ slice thickness (depending on scanner type). On scanners with the capacity for prospective triggering, studies are acquired with this technique whenever heart rate is adequately controlled $(\mathrm{HR}<65)$ and regular. For retrospective acquisitions, dose modulation is used to minimise radiation dose.

Standard initial reconstruction of the coronary CTA data set is performed at $75 \%$ of the RR interval, with additional reconstructions performed as required for image interpretation. When retrospective gating is used, reconstructions for functional assessment are performed in 10 phases with $10 \%$ increments.

\section{Participating sites}

Participating centres have a 64 detector multidetector computed tomography (MDCT) or greater with the capacity to perform cardiac CT, an expert reader in cardiology or radiology (as defined by American Heart Association (AHA)/ American College of Cardiology (ACC) training standards or their international equivalent) and a strong collaboration between the imaging team and the clinical perioperative medicine service to facilitate patient recruitment. The participating sites are listed in the appendix. Each participating site submits several scans to confirm adherence to the study imaging protocol and achievement of adequate image quality prior to the initiation of study recruitment. 


\section{CTA interpretation and blinding}

Coronary CTA is read by an expert radiologist or cardiologist. These individuals read each coronary CTA exam without knowledge of the clinical data. They report findings for each vessel, in terms of nature of plaque and extent of narrowing, and they determine whether each of the following four findings are present on the coronary CTA: (1) normal-no evidence of coronary atherosclerotic plaque (this excludes subsequent findings), (2) non-obstructive CAD-evidence of at least one coronary artery plaque with a $<50 \%$ stenosis, (3) obstructive CAD - at least one coronary artery plaque with a $\geq 50 \%$ stenosis, or (4) obstructive plaque with high-risk anatomy ( $\geq 50 \%$ stenosis of the left main, $\geq 50 \%$ stenosis in three coronary arteries, or $\geq 50 \%$ stenosis in two coronary arteries including the proximal left anterior descending (LAD) artery). Where calcium and/or motion artefacts limit interpretability, the segments are identified as non-evaluable and a forced interpretation on the presence of stenosis is made. If there are more than four non-evaluable segments, the scan is considered non-diagnostic and excluded from the analysis.

The classification of CTA findings being used in CTA VISION predicts the risk of mortality over 1-2 years in patients with stable CAD in the non-operative setting, ${ }^{16}$ similar to the results of the well-validated prognostic classification of invasive coronary angiography that puts increased importance on the number of vessels with $\geq 50 \%$ stenoses, with particular emphasis on involvement of the proximal LAD. ${ }^{17}$ Assessment of stenosis severity into board categories (ie, $50-70 \%$ vs $\geq 70 \%$ ) demonstrates very good agreement between coronary CTA and invasive angiography $(\kappa=0.74) .{ }^{18}$ The reader calculates a calcium score using the Agatston method using the noncontrast scan. ${ }^{19}$ This score is recorded and is available for subsequent analyses.

If a patient is discovered to have a $\geq 50 \%$ stenosis of the left main we immediately provide this test result to the patient's physicians. All other patients have their results withheld from the clinical care team until 30 days after surgery.

\section{Patient follow-up}

After surgery, patients have a troponin measurement drawn 6-12 h after surgery and on the first, second and third days after surgery. Standard orders ensure that these tests are undertaken. Orders also ensure that an ECG is undertaken immediately after an elevated troponin measurement is detected. Patients who are discovered to have an elevated troponin with or without ECG changes will usually undergo an echocardiogram or a functional evaluation such as nuclear perfusion imaging or stress MRI. Where clinically indicated, invasive coronary angiography is performed.

Research personnel follow patients throughout their time in hospital and personally evaluate patients and review their medical records ensuring study orders have been followed and noting any primary or secondary outcomes. The research personnel contact patients by phone at 30 days and 1 year postsurgery. If patients indicate that they have experienced an outcome or hospitalisation, the research personnel contact their physicians to obtain the appropriate documentation.

\section{Study outcomes}

For our first objective (ie, to determine if preoperative coronary CTA has additional predictive value beyond clinical variables) our primary outcome is a major cardiac event (ie, a composite of cardiovascular death and non-fatal myocardial infarction) at 30 days postsurgery. Individual secondary outcomes for our first objective at 1 year after surgery include cardiovascular death, myocardial infarction and coronary revascularisation. For our second objective (ie, to determine the underlying coronary anatomy associated with perioperative myocardial infarction) our primary and only outcome is myocardial infarction at $\mathbf{3 0}$ days after surgery.

The first step in determining the underlying coronary artery anatomy associated with a perioperative myocardial infarction is to determine the region of the myocardial infarction using a combination of clinical and non-invasive tests. Two cardiologists blinded to the CTA results will independently determine this using a prespecified algorithm based on literature and expert opinion (table 1). After establishing this, we then look at what the expert CTA evaluators stated was the underlying coronary artery anatomy (eg, <50\% stenosis, 50$69 \%$ stenosis and $\geq 70 \%$ stenosis) in the coronary artery that supplied the region of the myocardial infarction.

\section{Outcome adjudication}

A committee of clinicians who are blinded to the CTA results adjudicate the outcomes of death and myocardial infarction. We will use the decisions from the Adjudication Committee for all statistical analyses.

\section{Sample size}

Our sample size calculation is based on our primary objective (ie, to determine if preoperative coronary CTA has additional predictive value beyond clinical variables). Of our two objectives, this objective requires the largest number of patients to ensure the stability of the prediction model. The VISION Study will determine the optimal clinical risk prediction model, and we will then undertake a multivariable analysis to determine if the coronary CTA results have additional predictive value beyond the VISION clinical risk prediction model. Simulation studies demonstrate that logistic models require 12-15 events per predictor to produce stable estimates. ${ }^{2021}$ We will evaluate four potential predictors in our multivariable analysis: one VISION clinical risk predication score and three types of coronary CTA results (ie, non-obstructive CAD, obstructive CAD and obstructive plaque with high-risk anatomy) with one reference category (normal). Given our eligibility criteria, 
Table 1 Diagnostic confidence in determination of culprit lesion in patients who fulfil our definition of myocardial infarction

\begin{tabular}{ll}
\hline Diagnostic confidence & Criteria \\
\hline Highly probable location of & Thrombus or features of recent plaque rupture (irregular margins, hazy appearance and \\
lesion & dissection) coronary plaque fissure seen on invasive angiography or \\
Probable location of lesion & New area of infarction on cardiac MRI \\
& New wall motion abnormality (as determined through comparison of preoperative and \\
& postoperative echocardiography or MRI \\
& New wall motion abnormality as determined through comparison of preoperative CTA \\
& myocardial function and postoperative echocardiography or \\
Possible location of lesion & New waves in two contiguous leads on the patients ECG \\
& ST segment elevation ( $\geq 2$ mm in leads $\mathrm{V}_{1}$, $\mathrm{V}_{2}$ or $\mathrm{V}_{3}$ OR $\geq 1$ mm in the other leads) in two \\
& contiguous leads \\
& ST segment depression $(\geq 1 \mathrm{~mm})$ in two contiguous leads \\
& Symmetric inversion of $\mathrm{T}$ waves $\geq 1 \mathrm{~mm}$ in at least two contiguous leads or \\
& Presumed new cardiac wall motion abnormality on echocardiography \\
& Presumed new fixed defect on $\mathrm{SPECT}$ testing
\end{tabular}

we expect that the study participants will be evenly distributed across the four CTA result groups.

On the basis of the VISION Pilot Study and a previous non-invasive cardiac testing study that we undertook in a similar surgical population, ${ }^{22}$ we expect a $6 \%$ event rate for major perioperative cardiac events in this study. table 2 presents the various sample sizes needed to test four variables in a multivariable analysis based upon various event rates and the required number of events per variable. As the table indicates, if our event rate is $6 \%$ we will need 1000 patients to achieve stable estimates. If our event rate is $4 \%$, we may need up to 1500 patients. We are targeting a sample size of 1500 patients but this may change depending on our event rate at 1000 patients.

\section{Data analysis}

To address our primary objective, we will undertake a multivariable logistic regression analysis in which the dependent variable is a major perioperative cardiac event at 30 days after surgery and the independent

Table 2 Sample size needed to test four variables in a multivariable analysis based upon various event rates and the required number of events per variable

\begin{tabular}{|c|c|c|c|}
\hline \multirow{2}{*}{$\begin{array}{l}\text { Required } \\
\text { number } \\
\text { of events } \\
\text { per variable } \\
\end{array}$} & \multirow{2}{*}{$\begin{array}{l}\text { Number of } \\
\text { events } \\
\text { needed }\end{array}$} & \multicolumn{2}{|c|}{$\begin{array}{l}\text { Sample size needed to } \\
\text { test four variables in a } \\
\text { multivariable analysis } \\
\text { based on various event } \\
\text { rates }\end{array}$} \\
\hline & & $4 \%$ & $6 \%$ \\
\hline 10 & 40 & 1000 & 667 \\
\hline 12 & 48 & 1200 & 800 \\
\hline 15 & 60 & 1500 & 1000 \\
\hline
\end{tabular}

variables are the VISION clinical risk prediction score and the four coronary CTA results discussed above. For this logistic regression analysis we will use forced simultaneous entry (all candidate variables will remain in the model) as opposed to automated stepwise selection, because simulation studies have demonstrated a higher risk of overfitting with the latter approach. ${ }^{23} 24$ To assess the reliability of our models we will undertake bootstrapping, ${ }^{25}$ because this technique is superior to crossvalidation and jack-knife techniques. ${ }^{26}$ We will test the hypothesis that coronary CTA will have additional predictive value, beyond clinical variables, for the occurrence of major perioperative cardiac events in patients undergoing non-cardiac surgery using the likelihood ratio (LR) test: $\mathrm{LR}$ test $=-2 \ln (\mathrm{L} 1 / \mathrm{L} 2)$ where $\mathrm{L} 1$ and $\mathrm{L} 2$ are the likelihood for the reduced model with VISION score alone, and likelihood for the full model with both VISION score and CTA variables. ${ }^{27}$

For the logistic model we will report the ORs, 95\% CIs, and associated $\mathrm{p}$ values. For all tests, we will use $\alpha=0.05$ level of significance. For all significant associations we will report the likelihood ratio and the $95 \%$ CI.

Examination of residuals will provide an assessment of model assumptions for regression analyses. Goodness-of-fit for the models will be performed using appropriate Hosmer-Lemeshov tests. For the multivariable regression analysis, multicolinearity (correlations among predictor variables) may exist. ${ }^{28}$ We will assess colinearity using the variance inflation factor (VIF) which measures the extent to which the variance of the model coefficients will be inflated (because of the correlation of the variable with other predictor variables) if that variable is included in the model. We will consider variables with VIF $>10$ colinear and we will exclude one of these variables from the analysis. ${ }^{29}$ 
For our secondary objective we will determine the proportion of patients suffering a perioperative myocardial infarction who on their coronary CTA the myocardial infarction associated artery had a coronary artery stenosis of $<50 \%, 50-69 \%, \geq 70-99 \%, 100 \%$ or no coronary artery stenosis and the associated 95\% CIs. We will perform all analyses using SAS version 9.2 (Cary, North Carolina, USA).

\section{Ethics}

Coronary CTA results in a measurable radiation exposure. Volume coverage of the whole heart using standard acquisition parameters will approximately result in an effective dose of $10-12 \mathrm{mSv}$ if retrospectively gated CTA is performed. The dose will likely be substantially lower if prospectively triggered CTA is performed. The maximum anticipated does is similar to a nuclear perfusion scan $(8-12 \mathrm{mSv})$, but greater than a standard chest CT $(5-7 \mathrm{mSv})^{30}$ and equals 3-4 years of the annual average effective dose from background radiation (3.6 mSv/year) or approximately $20 \%$ of the annual whole body effective dose that is allowed for a radiation worker (radiologist, radiological technologist; $50 \mathrm{mSv}$ / year). ${ }^{31}$

We feel that blinding of treating physicians to the coronary CTA findings is important to provide the most unbiased assessment of its prognostic capabilities. Thus, in keeping with prior studies that evaluated non-invasive tests in patients undergoing vascular surgery, ${ }^{5} 32$ the attending surgeons and consultants in our study will not know the results of the preoperative coronary CTA. The best evidence currently available from the CARP Trial $^{33}$ and DECREASE-V Trial ${ }^{34}$ suggests that there is no benefit to prophylactic coronary revascularisation prior to non-cardiac surgery and therefore, the CTA results are not required to guide treatment prior to non-cardiac surgery. In the CARP Trial one-third of the patients had three vessel CAD, ${ }^{33}$ and in the DECREASE-V Trial $67 \%$ of the patients had three vessel CAD. ${ }^{34}$ The only exception is haemodynamically significant left main disease which was excluded in the CARP trial. Thus, if the coronary CTA suggests significant left main disease, the results will be immediately disclosed to the treating physicians. For all patients in the study, the results of coronary CTA will be provided to all family physicians, internal medicine and cardiology consultants involved in the care of the patients at 30 days postsurgery.

An external safety and monitoring committee (ESMC) will convene early in the study and will meet again at regular intervals. Interim analyses will be conducted when approximately $25 \%, 50 \%$ and $75 \%$ of the expected events have occurred and the data are available. The analyses will be conducted on the total of adjudicated and unadjudicated events at the appropriate time points. If the ESMC decides that a definitive conclusion has been reached for the overall study population or a specific subgroup, they will immediately unblind the
Co-Principal Investigators and discuss the results together.

\section{CONCLUSION}

Coronary CTA is a novel application of CT scanning with potentially important clinical applications. This study will evaluate the role of coronary CTA in risk stratification prior to non-cardiac surgery. If we demonstrate that CTA has important additional predictive value beyond clinical information in patients undergoing elective noncardiac surgery, this finding would allow this test to facilitate informed patient decision-making about the risks of surgery and guide perioperative patient management. This study will also provide insights into the underlying coronary anatomy of coronary arteries that cause myocardial infarction in the perioperative setting. This knowledge will inform the selection of targeted prevention and management interventions to evaluate in large perioperative randomised controlled trials. Considering that over 200 million adults undergo major non-cardiac surgery annually and that we know little about how to predict or manage major perioperative cardiac events, highlights the importance of the Coronary CTA VISION Study.

\section{Author affiliations}

${ }^{1}$ Department of Medicine, McMaster University, Hamilton, Ontario, Canada

${ }^{2}$ Division of Cardiology, University of Alberta, Edmonton, Alberta, Canada

${ }^{3}$ Department of Medicine (Cardiology) and Radiology, University of Ottawa Heart Institute, Ottawa, Ontario, Canada

${ }^{4}$ Department of Anesthesia and Intensive Care, The Chinese University of Hong Kong, Prince of Wales Hospital, Shatin, Hong Kong

${ }^{5}$ Departments of Radiology, Inokusi Hospital, Durban, South Africa

${ }^{6}$ Division of Clinical and Translational Research, Department of

Anesthesiology, Washington University, St. Louis, Washington, USA

${ }^{7}$ Department of Radiology, McMaster University, Hamilton, Ontario, Canada

${ }^{8}$ Department of Anesthesia, Inokusi Hospital, Durban, South Africa

${ }^{9}$ Division of Radiology, Washington University, St. Louis, Washington, USA

${ }^{10}$ Division of Cardiology, University Health Network, Toronto, Ontario, Canada

${ }^{11}$ Department of Biomedical Imaging, University Malaya Research Imaging

Centre, Kuala Lumpur, Malaysia

${ }^{12}$ Division of Cardiology, All India Institute of Medical Sciences, New Delhi, India

${ }^{13}$ Department of Cardiology, Westmead Hospital \& The George Institute for

Global Health, The University of Sydney, Sydney, Australia

${ }^{14}$ Departments of Internal Medicine, Jagiellonian University Medical College, Krakow, Poland

${ }^{15}$ Division of General Internal Medicine, University of Western Ontario, London, Ontario, Canada

${ }^{16}$ Endovascular and Vascular Surgery Department, Cleveland Clinic

Foundation, Cleveland, Ohio, USA

${ }^{17}$ Department of Clinical Epidemiology and Biostatistics, McMaster University, Hamilton, Ontario, Canada

Contributors All authors provided intellectual input into the editing of the manuscript and preparation for publication.

Funding This work is supported by grants from Canadian Institutes of Health Research, the Hamilton Health Sciences New Investigator Fund, the McMaster University Division of Cardiology, the University of Western Ontario Department of Radiology and the University of Western Ontario Division of General Internal Medicine. The study funders have no role in study design, data collection, data analysis and interpretation, drafting of manuscripts or the decision to submit the report for publication. 
Competing interests None.

Provenance and peer review Not commissioned; internally peer reviewed.

\section{REFERENCES}

1. Devereaux PJ, Chan M, Eikelboom J. Major vascular complications in patients undergoing noncardiac surgery: the magnitude of the problem, risk prediction, surveillance, and prevention. In: Yusuf $S$, Cairns JA, Camm AJ, Fallen EL, Gersh BJ, eds. Evidence based cardiology. 3rd edn. London, England: BMJ Books, 2009:47-62.

2. Devereaux PJ, Yang H, Yusuf S, et al. Effects of extended-release metoprolol succinate in patients undergoing non-cardiac surgery (POISE trial): a randomized controlled trial. Lancet 2008;371:1839-47.

3. Devereaux PJ, Goldman L, Cook DJ, et al. Perioperative cardiac events in patients undergoing noncardiac surgery: a review of the magnitude of the problem, the pathophysiology of the events and methods to estimate and communicate risk. CMAJ 2005;173:627-34.

4. Beattie WS, Abdelnaem E, Wijeysundera DN, et al. A meta-analytic comparison of preoperative stress echocardiography and nuclear scintigraphy imaging. Anesth Analg 2006;102:8-16.

5. Vanzetto $G$, Machecourt J, Blendea $D$, et al. Additive value of thallium single-photon emission computed tomography myocardia imaging for prediction of perioperative events in clinically selected high cardiac risk patients having abdominal aortic surgery. $A m \mathrm{~J}$ Cardiol 1996;77:143-8.

6. Coley CM, Field TS, Abraham SA, et al. Usefulness of dipyridamole-thallium scanning for preoperative evaluation of cardiac risk for nonvascular surgery. Am J Cardiol 1992;69:1280-5.

7. Brown KA, Rowen M. Extent of jeopardized viable myocardium determined by myocardial perfusion imaging best predicts perioperative cardiac events in patients undergoing noncardiac surgery. J Am Coll Cardiol 1993;21:325-30.

8. Boersma E, Poldermans D, Bax JJ, et al. Predictors of cardiac events after major vascular surgery: role of clinical characteristics, dobutamine echocardiography, and beta-blocker therapy. JAMA 2001;285:1865-73.

9. Poldermans D, Arnese M, Fioretti PM, et al. Improved cardiac risk stratification in major vascular surgery with dobutamine-atropine stress echocardiography. J Am Coll Cardiol 1995;26:648-53.

10. Mollet NR, Cademartiri F, Krestin GP, et al. Improved diagnostic accuracy with 16-row multi-slice computed tomography coronary angiography. J Am Coll Cardiol 2005;45:128-32.

11. Raff GL, Gallagher MJ, O'Neill WW, et al. Diagnostic accuracy of noninvasive coronary angiography using 64-slice spiral computed tomography. J Am Coll Cardiol 2005;46:552-7.

12. Leschka S, Alkadhi H, Plass A, et al. Accuracy of MSCT coronary angiography with 64-slice technology: first experience. Eur Heart 2005;26:1482-7.

13. Sheth T, Amlani S, Ellins ML, et al. CT coronary angiographic assessment of high risk coronary anatomy in patients with suspected coronary artery disease and intermediate pre-test probability. Am Heart J 2008;155:918-23.

14. Butler J, Shapiro M, Reiber J, et al. Extent and distribution of coronary artery disease: a comparative study of invasive versus noninvasive angiography with computed angiography. Am Heart $J$ 2007;153:378-84.

15. Effects of extended-release metoprolol succinate in patients undergoing non-cardiac surgery (POISE trial): a randomized controlled trial. Lancet 2008;371:1839-47.

16. Min JK, Shaw LJ, Devereux RB, et al. Prognostic value of multidetector coronary computed tomographic angiography for prediction of all-cause mortality. J Am Coll Cardiol 2007;50:1161-70.

17. Califf RM, Phillips HR 3rd, Hindman MC, et al. Prognostic value of a coronary artery jeopardy score. J Am Coll Cardiol 1985;5:1055-63.

18. Cury RC, Ferencik M, Achenbach S, et al. Accuracy of 16-slice multi-detector CT to quantify the degree of coronary artery stenosis: assessment of cross-sectional and longitudinal vessel reconstructions. Eur J Radiol 2006;57:345-50.

19. Agatston AS, Janowitz WR, Hildner FJ, et al. Quantification of coronary artery calcium using ultrafast computed tomography. J Am Coll Cardiol 1990;15:827-32.
20. Babyak MA. What you see may not be what you get: a brief, nontechnical introduction to overfitting in regression-type models. Psychosom Med 2004;66:411-21.

21. Peduzzi P, Concato J, Kemper E, et al. A simulation study of the number of events per variable in logistic regression analysis. J Clin Epidemiol 1996:49:1373-9.

22. Gulenchyn KY, Montgomery AG, Sumner G, et al. A pilot study evaluating the predictive value of noninvasive pharmacological cardiovascular stress testing before orthopaedic surgery. [Abstract] J Nucl Cardiol 2007;14:S3

23. Derksen S, Keselman H. Backward, forward and stepwise automated subset selection algorithms: frequency of obtaining authentic and noise variables. Br J Math Stat Psychol 1992;45:265-82.

24. Steyerberg EW, Eijkemans MJ, Harrell FE Jr., et al. Prognostic modeling with logistic regression analysis: in search of a sensible strategy in small data sets. Med Decis Making 2001;21:45-56.

25. Efron B, Tibshirani R. An introduction to the bootstrap. London Chapman \& Hall, 2003

26. Gong G. Cross-validation, the jackknife and the bootstrap: excess error estimation in forward logistic regression. J Am Stat Assoc 1986;81:108-13.

27. Kleinbaum D, Klein M. Logistic regression. 2nd edn. New York: Springer-Verlag, 2002.

28. Wax Y. Collinearity diagnosis for a relative risk regression analysis: an application to assessment of diet-cancer relationship in epidemiological studies. Stat Med 1992;11:1273-87.

29. Kline R. Data preparation and screening. In: Kline R, ed. Principles and practice of structural equation modeling. New York: The Guilford Press, 1998:67-94.

30. Hunold P, Vogt FM, Schmermund A, et al. Radiation exposure during cardiac CT: effective doses at multi-detector row CT and electron-beam CT. Radiology 2003;226:145-52

31. Jakobs TF, Becker CR, Ohnesorge B, et al. Multislice helical CT of the heart with retrospective ECG gating: reduction of radiation exposure by ECG-controlled tube current modulation. Eur Radiol 2002;12:1081-6.

32. de Virgilio C, Toosie K, Elbassir M, et al. Dipyridamole-thallium/ sestamibi before vascular surgery: a prospective blinded study in moderate-risk patients. J Vasc Surg 2000;32:77-89.

33. McFalls EO, Ward HB, Moritz TE, et al. Coronary-artery revascularization before elective major vascular surgery. $N$ Engl J Med 2004;351:2795-804.

34. Poldermans D, Schouten O, Vidakovic R, et al. A clinical randomized trial to evaluate the safety of a noninvasive approach in high-risk patients undergoing major vascular surgery: the DECREASE-V Pilot Study. J Am Coll Cardiol 2007;49:1763-9.

\section{APPENDIX}

Co-Principal Investigators: T Sheth, P J Devereaux

Co-ordinating Centre: S Pettit, E Wendland, D Heels-Ansdell

External Safety and Monitoring Committee: V Montori (Chair), $\mathrm{N}$ Valettas, K Thorlund

\section{Investigators}

Hamilton, Canada: L Stewart, V Tandon, M Ferri

Ottawa, Canada: B Chow, P Jetty

Edmonton, Canada: C Butler, R Coulden, M Graham, M Jacka

Hong Kong, China: M Chan, GYS Choi

Durban, South Africa: A Mitha, T Kisten, B Biccard, R Rodseth

St. Louis, USA: P Nagele, P Woodard

Toronto, Canada: A Crean

Kuala Lampur, Malaysia: C Wang, Y F Abdul Aziz, R Sandhu

Sydney, Australia: C K Chow

New Delhi, India: G Karthikeyan, G Gulati

London, Canada: M Markobrada, A Islam, A Goela

Krakow, Poland: W Szczeklik, T Miszalski-Jamka, B Sokolowska

Cleveland, USA: T Mastracci, M Desai 